\title{
Research and Design of Multimedia NSS for E-Commerce
}

\author{
Hanxiao Shi , Guiyi Wei \\ Computer and Information Engineering College, Zhejiang Gongshang \\ University, Hangzhou, 310035, China \\ hxshi@mail.zjgsu.edu.cn
}

\begin{abstract}
As the Internet has become mainstream, so has Electronic commerce (EC) become an important part of conducting business. NSS facing EC will be a very important subsystem during the process of EC development. This paper introduces the current research situation about NSS, stresses on the whole system architecture and key technologies, sets forth solution scheme about some important problem, such as multiparty cooperation text technology and inter-stream synchronization. Our goal is to develop a NSS prototype and to test its feasibility in simulation environment. It also creates a user-friendly interface for Graphic User's Interface (GUI). The implementation prototype system demonstrates excellent performance.
\end{abstract}

\section{Introduction}

With the great development of Internet and EC, trade mode will have a thorough change. A business transaction consists of three main phases, namely searching for potential business partners, negotiating about contract conditions and finalizing the business contract, and fulfilling the contract. Negotiation in traditional as well as in electronic commerce is a complex communication process. In the networking environment, the process of business negotiation will be an online mode in stead of the traditional face-to-face mode. The research on EC-oriented negotiation support system (NSS) is an aid for EC, as well as a new explorer for the development of NSS. This project is very important in the domain of EC and related business.

Apparently, improvement of network infrastructure is a base to implement online NSS. We propose an interaction platform over audio-video based on multimedia technology, and present a function mode of multiparty cooperation text technology (MCTT) and third party authority technology, which can realize effective E-Contract. Otherwise, take advantage of NSS, to give better service to any negotiators. As a result, the research of EC-oriented NSS can realize visible negotiations in different place. In a word, it saves the negotiations cost, enhances the negotiations efficiency.

Please use the following format when citing this chapter:

Shi, II., Wei, G., 2007, in IFIP International Federation for Information Processing, Volume 251, Integration and Innovation Orient to E-Socicly Volume1, Wang, W. (Eds), (Boston: Springer), pp. 126-133. 
This paper has a deep research on multimedia technology, MCTT, negotiation server design and so on, proposes a whole negotiation flow structure, implements a multimedia-based NSS.

\section{Related Work}

Negotiation is a decision process in which two or more parties make individual decisions and interact with each other for mutual gain. NSS has introduced advanced computer-information technology and decision support system (DSS) theory into the domain of negotiation collision; it provides some question-solution tools [1-3] for any negotiators through many sides of technology and theory, such as computerhuman interaction, operation research, DSS, behavioral science, psychology, and so on.

In general, NSSs have the following basic features[4]: (1) a formalism to describe the negotiation activity in terms of choices and outcomes, (2) a way to generally characterize the associated outcome probabilities, and (3) a methodology for processing the model to evaluate the expected values of choice alternatives. NSSs normally assist negotiators to assess situations, generate and evaluate options, and implement decisions. However, most NSSs do not consider the generation of contracts, which we consider to be the primary aim of negotiation in ecommerce, as an outcome of negotiation process. For example, NEGOTIATOR [5] seeks to guide negotiators to move their individual goals and judgments to enhance the chance of achieving a common solution. It supports problem adaptation through information sharing, concession making, and problem restructuring. However, NEGOTIATOR only helps the negotiators make decisions without any support to other entities involved in negotiation, such as contracts. INSPIRE (InterNeg Support Program for Intercultural Research) [2] is a Web-based prototype for supporting inter-cultural as well as intra-cultural negotiations. It can conduct negotiation anonymously, evaluate the goodness of an offer, and review the history of a negotiation. INSPIRE supports the communication among negotiators by exchanging messages, but we propose direct interactions among different entities with Web services.

Recently, researchers are developing negotiation protocols for agent automation in e-commerce environments. Bartolini et al. [6] develop an agent-based software framework for automated negotiation, aiming for reuse. Tamma et al. [7] further propose negotiation protocols to be expressed in terms of shared ontology among agents. However, they only showed an example protocol for English auction in the paper with rule-based axioms and did not detail their negotiation process support and management environment. Neither did they present Web services implementation frameworks that support both human and programmatic users as in this paper. Griffel et al. [8] present an application of contract negotiation by mobile agents. A contract is represented as an object that can be accessed by the negotiation parties. Each party has the opportunity to change or insert clauses. They only provide a conceptual view of their approach, without any supporting formal model.

Gradually NSS based on web and E-commerce become to a research focus, there are not a few researches on this domain. References $[2,9$, and 10] show CBSS and 
INSPIRE (Carleton University) which have referred to some related researches. These references propose some specifics about these NSS: (1) Provide a simultaneous distribution negotiation; (2) Have a real-time multiparty interaction; (3) Provide a structural negotiation procedure; (4) Provide some security functions, such as identity recognition, data secrecy. Reference [11] proposes an evolution computation method in electronic business negotiation, which makes use of negotiation agents to select a best negotiation strategy from strategy-base according to enterprise targets and benefits.

Reference [12] proposes a NSS model based on B2B mode - Negoisst, which supports negotiators to do complicated E-Negotiation. It has three main functions: auto-questioning, intelligent negotiation agents, negotiation support. Multinegotiation and file management are implemented by communication technology and information system.

\section{Analysis and Design of Multimedia NSS}

\subsection{Work Flow of Negotiation Support Systems}

Negotiation support systems do not automate the negotiation process but provide IT support for complex negotiations, leaving the control over the negotiation process with the human negotiators. There are several participators during a negotiation, and each of them is a decision maker, they analyze and judge the situation of negotiation, and make decisions. From the decision-supporting view, the process of negotiation can be considered as a decision-making process of negotiators, and it can be divided into 4 stages: conceptualizing negotiating problem; expressing negotiating problem; problem solving; analyzing the solving plan. Negotiating process starts from conceptualize negotiating problem till analyzing the solving plan in theory, and go along step by step. However, the feedback and circle in fact is much more complex than this, during which multimedia and cooperation text technology are used. During the process of negotiation, participators reach an agreement on problems need to negotiate, and this is a necessity to farther the negotiation. The negotiate model is shown in Figure. 1. 


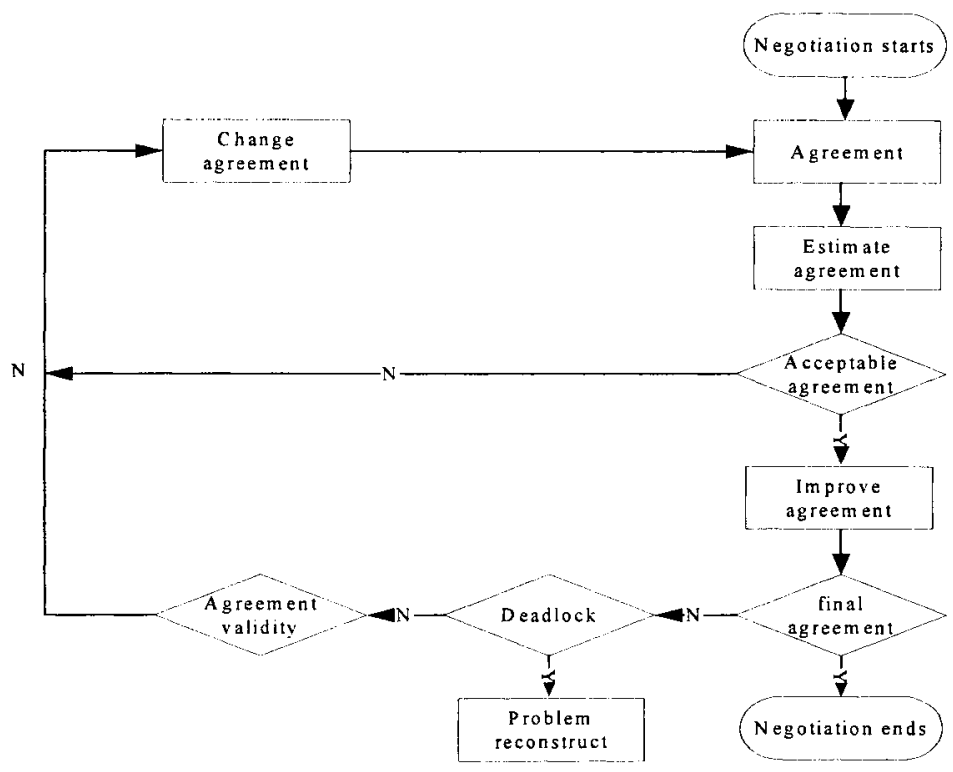

Figure. 3. A negotiation system model

\subsection{Function Models of NSS}

This is a net-based NSS, written in Java and implemented in a client - server architecture. This system contains several function models as follows:

(1) User management model: including user registration, user information modification, etc

(2) Client to client audio and video interaction model: simulating face to face communication, and all this data transfer without the server.

(3) Character interaction model: supplying traditional network communicating tool, and keep all the interaction information on the server if needed.

(4) Cooperation text edit model: realizing online user's synchronous and asynchronous data interchange.

(5) Data communication management model: supporting all the communications between clients (negotiators) and server.

(6) E-contract management model: after editing and conforming the cooperation text, an e-contract will be created based on this. Realizing e-contract interchange on Internet.

\subsection{System Architecture}

NSS facing EC will be a very important subsystem during the process of EC development, the client access the website by web browser, download the client of NSS, and connect the server after installing. Besides, the system supply mutual supports with some models like Internet information service system, electronic 
identity identification system, electronic payment system, and generate e-contract as a part of EC archives, to realize e-contract interchange on Internet. The web part adopts the B/S structure, and the NSS part adopts the C/S structure.

The whole working model is shown in Figure. 2.

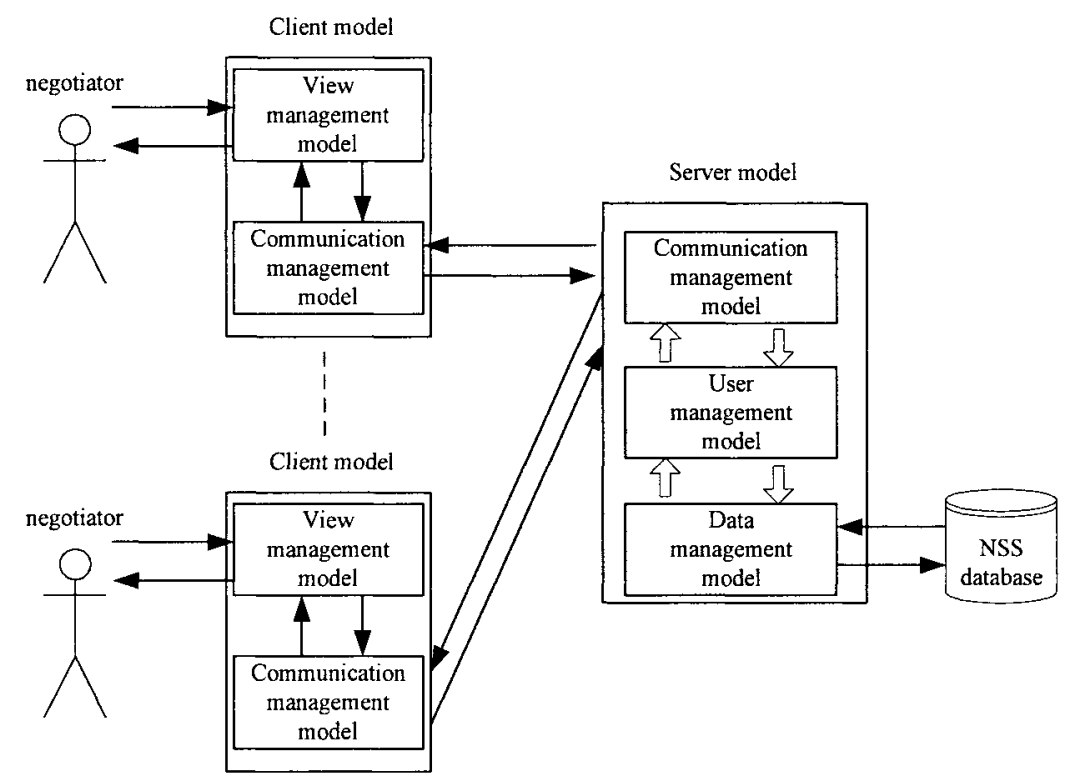

Figure. 2. System architecture of NSS

At the client side, system can be divided into two models: communication management and view expression; At the server side, system includes communication management model, user management model, and data management model. The communication management model is responsible for the information interchange between client and server, and the key part of it is a communication protocol, through this protocol, the data flow between client and server fulfill all kinds of functions like encode and decode, complete control orders, transfer data. The user management model plays a center role in supervising communication models and harmonizing the information interchanging between different users. The data management model is responsible for the data interchange between database, and reading, storing, $\mathrm{SQL}$ querying etc. 


\section{Key Technologies of System}

\subsection{Multiparty Cooperation Text Technology}

Real-time cooperation text editing system (like Grove [13] and Reduce [14]) is a very important part during the applications of computer supported cooperative work (CSCW), it allows a group of cooperators from different places to edit shared files at the same time. It has such characteristics as (1) rapid response; (2) distribution; (3) highly concurrent; (4) consistency. In order to reach the goal of rapid response, fullcopy structure is frequently used to deal with shared files in cooperation editing system, which means copy shared files to every participator. This structure allows operations to be executed locally, and then spread to other participator, the response time is very short. However, some problems may be raised under this structure, an arithmetic of concurrent control is needed to keep the consistency of files.

This system adopt mark method to realize MCTT [15], through marking the shared files, it will hide the changed part, no matter how many operations have been executed before. This assures the file under operation is exactly the same one the moment before the operation works, to realize the maintenance of operation process

\subsection{Synchronization of Audio and Video Stream}

A system supporting audio and video communication based on $\mathrm{H} .323$ protocol. After collecting and encoding, audio and video data arrive the receiver through Internet, and store to each play-buffer after decoding. The inter-stream synchronization is controlled by media synchronization between these buffers.

During a multimedia system, there are many media streams, the independent stream is named main-media stream; and during playing, those who depend on this stream are named sub-media stream. Main-media stream plays at a normal speed, to keep synchronization, the sub-media stream may need to jump or pause. During multimedia communication system, considering our ear is relatively more sensitive to sound pause, repeat and playing rate adjustment, audio stream is usually regarded as the main-media stream, and other streams including video are treated as the submedia stream, to realize the synchronization by adjusting playing time. As the mainmedia stream, audio adopts G.723.1 encode standard, and the sub-media stream, video adopts H.263 encode standard. When the Internet is unobstructed, it can assure the video and audio stream transfer with the settled rapid, the delay is basically fixed, the jitter is small, and the space between audio and video in both sender and receiver keeps the same: that means media data are almost integrated. When the Internet is blocked, the inter-media asynchronism may exist, and some technologies must be taken to realize the synchronization. In this system, time stamp in RTP package head is used to solve this problem. The detail algorithm is aimed at optimizing and dealing with sub-media stream (mainly video data) comparing with the main-media stream. Tv: the time stamp of video frame arrived, Ta: the time stamp of audio frame now, Td: the tolerance of audio frame asynchronism. 


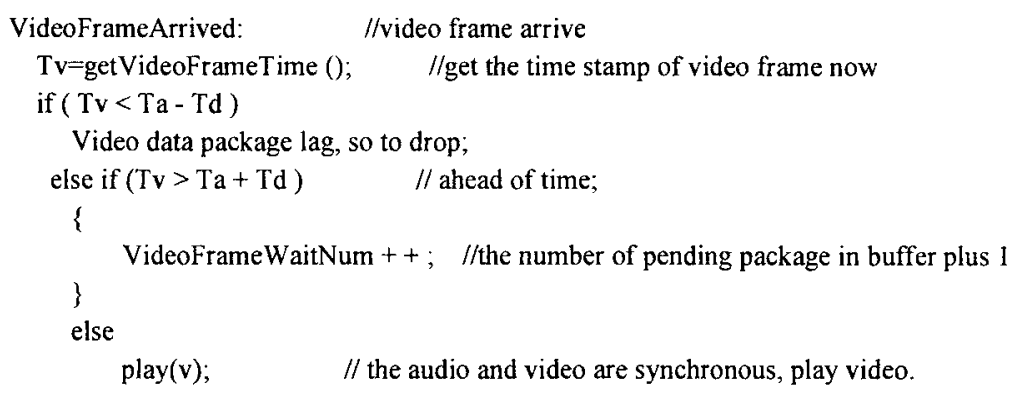

The process of audio stream is relatively simple, as soon as audio data arrive, the audio device plays them as well as system needs to check video data. If video frame is not null at same time, video data is played as mentioned above.

\section{Summaries}

In this paper, we have introduced the concept and development of NSS through laying out some representative works and some researches on NSS. We have also pointed out their respective merits and shortcomings based on comparison and analysis. In the meanwhile, we have designed a NSS's work flow and some function models. At last, we have brought forward the system realization architecture of NSS, and discussed some key technologies, such as multiparty cooperation text technology, inter-media synchronization, and so on. By simulating experiment in intranet, this system realized online-negotiation with multimedia integration, offering each negotiator a kind interface and multi-choices of assistant function. And after a period of testing, this system works well. In some degree, it meets the real-time need of NSS, and has some important significance in promoting the development of china EC.

\section{Acknowledgement}

The authors wish to thank the support of Zhejiang Provincial Education Department for Scientific Research Fund under grant Number 20061078 and the Natural Science Foundation of Zhejiang Province, P. R. China for the National Science Fund under grant Number Y105356. We would also like to thank our referees for their helpful comments and suggestions.

\section{References}

1. M.M. Delaney., "An Empirical Study of the Efficacy of A Computerized Negotiation Support System (NSS)", Decision Support Systems, 20(3), 185-197 (1997). 
G.E. Kersten and S.J. Noronha, WWW-based Negotiation Support: Design, Implementation and Use, Decision Support Systems, 25(2), 135-154 (1999).

3. Y.W.S. Stanley, C.B. Huang and J. Hammer., A Replicable Web-Based Negotiation Server for E-Commerce, IEEE Thirty-third Hawaii International Conference on System Sciences (HICSS-33), 212-221 (2000).

4. InterNeg, For and about Negotiations (2000), http://interneg.carleton.ca.

T.X. Bui and M.F. Shakun, Negotiation Processes, Evolutionary Systems Design, and Negotiator, Group Decision and Negotiation, 5, 339- 353 (1996).

5. C. Bartolini, C. Preist, and N.R. Jennings, Architecting for Reuse: A Software Framework for Automated Negotiation, Proceedings of the 3rd International Workshop on AgentOriented Software Engineering, 87-98 (2002).

6. V. Tamma, M. Wooldridge and I. Dickinson, An Ontology-Based Approach to Automated Negotiation, Proceedings of the Fourth International Workshop on Agent-Mediated Elctronic Commerce (AMEC-2002), 219-237 (2002).

7. F. Griffel, M.T. Tu, M. Münke, M.M. da Silva, M. Merz. and W. Lamersdorf, Electronic Contract Negotiation as An Application Niche for Mobile Agents, Proceedings of the First International Enterprise Distributed Object Computing, 354 - 365 (1997).

8.G.E Kerstern, Negotiation Support Systems and Negotiation Agents, Modèles et Systèmes Multi-Agents pour la Gestion de l'Environement et des Territoires, Cemagref ENGREF, Clermont-Ferrand, France, 307-316 (1998).

9. Y. Yuan, R.B. Suarga and N. Archer, A Web-Based Negotiation Support System, International journal of electronic markets, 8(3), 13-17 (1998).

10.Y.A. Salem and A.A. Al-Fahoum, An Evolutionary Computation Approach to Electricity Trade Negotiation, Advances in Engineering Software, 36(3), 173-179(2005).

11.M. Schoop, A. Jertila and T. List, Negoisst:, A Negotiation Support System for Electronic Business-to-Business Negotiations in E-Commerce, Data and Knowledge Engineering, 47(3), 371-401 (2003).

12. C.A. Ellis and S.J. Gibbs, Concurrency Control in Groupware Systems, Proceedings of 19th ACM SIGMOD Conference of Management of Data, 399- 407 (1989).

13.C. Sun, X. Jia, Y. Zhang and D. Chen, Achieving Convergence, Causality-Preservation, and Intention-Preservation in. Real-Time Cooperative Editing Systems, ACM Transactions on Computer-Human Interaction, 5(1), 63-108 (1998).

14. X.Y Wu and N. Gu, A Concurrency Control Method Based on Document Marking, Journal of Computer Research and Development, 39(12), 1663-1667 (2002). 